\title{
Bayesian inference on reliability in a multicomponent stress-strength bathtub-shaped model based on record values
}

\author{
Abbas Pak \\ Department of Computer Sciences, Faculty of Mathematical Sciences, \\ Shahrekord University, Iran \\ abbas.pak1982@gmail.com \\ Nayereh Bagheri Khoolenjani \\ Department of Statistics, University of Isfahan, Isfahan, Iran \\ n.b.khoolenjani@gmail.com \\ Manoj Kumar Rastogi \\ Department of Statistics, Patna University, Patna, India. \\ manojlogin@gmail.com
}

\begin{abstract}
In the literature, there are a well-developed estimation techniques for the reliability assessment in multicomponent stress-strength models when the information about all the experimental units are available. However, in real applications, only observations that exceed (or fall below) the current value may be recorded. In this paper, assuming that the components of the system follow bathtub-shaped distribution, we investigate Bayesian estimation of the reliability of a multicomponent stress-strength system when the available data are reported in terms of record values. Considering squared error, linex and entropy loss functions, various Bayes estimates of the reliability are derived. Because there are not closed forms for the Bayes estimates, we will use Lindley's method to calculate the approximate Bayes estimates. Further, for comparison purposes, the maximum likelihood estimate of the reliability parameter is obtained. Finally, simulation studies are conducted in order to evaluate the performances of the proposed procedures and analysis of real data sets is provided.
\end{abstract}

Keywords: Bathtub-shaped model, Maximum likelihood technique, Bayesian viewpoint, Stress-strength model.

\section{Introduction}

The bathtub-shaped model specified by the probability density function (pdf)

$$
f(z ; \gamma, \delta)=\gamma \delta z^{\delta-1} e^{z^{\delta}} e^{\gamma\left(1-e^{z^{\delta}}\right)}, \quad z>0, \quad \gamma, \delta>0,
$$

and survival function

$$
S(z ; \gamma, \delta)=e^{\gamma\left(1-e^{z^{\delta}}\right)}, \quad z>0, \quad \gamma, \delta>0,
$$

was investigated by Chen (2000) as a new distribution useful to analyze lifetime data. Several authors discussed on inferential techniques for this model based on different complete and censored samples; see for example Ahmed (2014) and Wu et al. (2005). From now on bathtub-shaped model will be denoted as $B S H(\gamma, \delta)$.

For many years, stress-strength models are well used in diverse areas such as quality control, engineering and medicine. In reliability engineering the parameter $R=P(X>Y)$ with the stress $Y$ and strength component $X$, shows the reliability of a system (Kotz et al. (2003)). Also, these models are used for comparison of two random variables. For example, comparison of capability of two workers, performances of two 
new products, treatment effects of two drugs. Several authors have used different inference techniques to estimate the reliability parameter $R$ based on various approaches and distributional assumptions on $(X, Y)$. Among others, Pak et al. (2014) studied nonBayesian and Bayesian estimation of the stress-strength parameter $R$ based on fuzzy observations. Estimation of $P(X>Y)$ in the models with correlated stress and strength is studied by Balakrishnan and Lai (2009). Inference for the stress-strength systems with bivariate Pareto model is studied by Hanagal (1997). Abd Elfattah and Marwa (2011) considered Bayesian estimation of stress-strength exponential model by using different loss functions. Makhdoom et al. (2016) derived Bayesian estimates of the reliability in stress-strength models with power Lindley components. Akgul and Senoglu (2017) have used ranked set sampling to derive Weibull sress-strength parameter.

Recently, several researchers pay attention to estimating the reliability in multicomponent stress-strength (MSS) models. In the MSS system there are $m$ identical and independent strength components and a common stress that functions when at least $r(1 \leq r \leq m)$ of the components survive. This MSS model is denoted as $r$-out-of- $m: G$ system. For example, consider an automobile with a V-8 engine that works if four cylinders are firing. So, it can be represented as 4-out-of-8: $G$ system. Another example may be a suspension bridge with $m$ pairs of vertical cables such that the bridge survives when at least $r$ number of vertical cables work. Inference on the reliability in MSS models when the stress and strength follow Weibull distribution is considered by Kizilaslan and Nadar (2015). Rao et al. (2012,2014) conducted a series of studies to estimate the reliability of MSS models by assuming generalized exponential and Burr XII distributions for the components. They have used classical approaches to compute the reliability estimation in $r$-out-of- $m: G$ models. Nadar and Kizilaslan (2016) considered Marshal-Olkin bivariate Weibull distribution and provided some inference procedures for MSS models. Dey et al. (2016) addressed MSS models consisting of Kumaraswamy distributed random variables.

The above inference for MSS models are derived when the complete information about all the involved units are available. However, in practice, we may deal with a record breaking data set in which only values larger (or smaller) than the current extreme value are reported. Using this type of data, we can save a lot in terms of cost and time. So, record data holds value in everyday life in the areas such as hydrology, sports, weather forecast, meteorological analysis, economics and lifetests. Due to the wide applications, there is vast literature on inference based on record data. An account on this topic is available in the excellent book of Arnold et al. (1998). Asgharzadeh et al. (2018) studied inference based on Lindley record data. Raqab et al. (2018) considered estimation of the two-parameter bathtub-shaped distribution based on record data. Pak and Dey (2019) discussed on statistical inference for the power Lindley model based on record values and inter-record times. Selim (2018) considered estimation and prediction for Nadarajah-Haghighi distribution based on record values. Dasylva (2018) developed Design-Based estimation with record-linked administrative files.

The objective of this paper is to provide Bayesian inferences on the reliability of $r$-out-of- $m$ : $G$ models by using upper record values. Such inferences are important in comparing two record processes. For example, we can use it to compare extreme environmental conditions in different months and check whether there are significant seasonal effects. Other examples include comparisons of extreme events in different areas and comparison of athletic abilities of men and women. Here, we assume that the strength 
and stress components are independent random variables distributed as bathtub-shaped model. First, maximum likelihood estimate (MLE) of the reliability parameter is derived. Then, considering various symmetric and asymmetric loss functions, some expressions are provided as the Bayes estimates of the reliability parameter. Since these expressions can not be simplified to nice closed forms, we employ Lindley's technique to obtain approximate Bayes estimates of the reliability.

The layout of this paper is as follows. Section 2 concerns ML estimation of the reliability parameter. Assuming squared error, linex and entropy loss functions, different Bayes estimates of the reliability are derived in Section 3. Simulation studies are conducted in Section 4 to assess the accuracy of the proposed methods and analysis of two real data sets is presented for illustrative purposes.

\section{Maximum likelihood estimation}

Suppose that the $m$ strength components of a MSS system are independent random variables (r.v.s) with the common cumulative distribution function (cdf) $G(x)$ and let $F(y)$ be the cdf of the stress r.v. $Y$. When the strength and stress components of the system follow $B S H\left(\gamma_{1}, \delta\right)$ and $B S H\left(\gamma_{2}, \delta\right)$, respectively, the reliability of MSS model can be obtained as

$$
\begin{aligned}
& R_{r, m}=\sum_{i=r}^{m}\left(\begin{array}{c}
m \\
i
\end{array}\right) \int_{0}^{\infty}(1-G(y))^{i}(G(y))^{m-i} d F(y) \\
& \left.=\sum_{i=r}^{m}\left(\begin{array}{c}
m \\
i
\end{array}\right) \int_{0}^{\infty} \gamma_{2} \delta y^{\delta-1} e^{y^{\delta}+i \gamma_{1}\left(1-e^{y^{\delta}}\right.}\right)\left(1-e^{\gamma_{1}\left(1-e^{y^{\delta}}\right)}\right)^{m-i} e^{\gamma_{2}\left(1-e^{y^{\delta}}\right)} d y \\
& =\sum_{i=r}^{m}\left(\begin{array}{c}
m \\
i
\end{array}\right) \gamma_{2} \int_{1}^{\infty} e^{\left(i \gamma_{1}+\gamma_{2}\right)(1-u)}\left(1-e^{\gamma_{1}(1-u)}\right)^{m-i} d y, \text { where } u=e^{y^{\delta}} .
\end{aligned}
$$

After simplification, the expression in (3) is expressed as

$$
\begin{aligned}
& R_{r, m}=\sum_{i=r}^{m} \sum_{j=0}^{m-i}\left(\begin{array}{c}
m \\
i
\end{array}\right)\left(\begin{array}{c}
m-i \\
j
\end{array}\right) \gamma_{2}(-1)^{j} e^{(i+j) \gamma_{1}+\gamma_{2}} \int_{1}^{\infty} e^{-u\left((i+j) \gamma_{1}+\gamma_{2}\right)} d y \\
& =\sum_{i=r}^{m} \sum_{j=0}^{m-i}(-1)^{j}\left(\begin{array}{c}
m \\
i
\end{array}\right)\left(\begin{array}{c}
m-i \\
j
\end{array}\right) \frac{\gamma_{2}}{(i+j) \gamma_{1}+\gamma_{2}} .
\end{aligned}
$$

For simplicity, the reliability parameter $R_{r, m}$ is hereafter referred to as $R$. Let $\left\{Z_{n}, n=1,2, \ldots\right\}$ be a sequence of identical and independent random variables. An observation $Z_{j}$ is called an upper record value if $Z_{j}>Z_{i}$ for all $i<j$. Considering this fact, the sequence $\left\{S_{n}, n \geq 1\right\}$ defined as: $S_{1}=1$ and $S_{n}=\min \left\{j: Z_{j}>Z_{S_{n-1}}\right\}, n \geq 2$ is called record time. Then, the sequence of upper record values is $\left\{X_{n}=Z_{S_{n}}, n=1,2, \ldots\right\}$.

Now assume that $\mathrm{u}=\left(u_{1}, \ldots, u_{n}\right)$ and $\mathrm{v}=\left(v_{1}, \ldots, v_{k}\right)$ be two sets of upper record values from $B S H\left(\gamma_{1}, \delta\right)$ and $B S H\left(\gamma_{2}, \delta\right)$, respectively. Then, the observed data likelihood function of $\gamma_{1}, \gamma_{2}$ and $\delta$ becomes 


$$
L_{O}\left(\gamma_{1}, \gamma_{2}, \delta ; \mathrm{u}, \mathrm{v}\right)=\gamma_{1}^{n} \gamma_{2}^{k} \delta^{n+k} e^{\sum_{i=1}^{n} u_{i}^{\delta}+\sum_{j=1}^{k} v_{j}^{\delta}} e^{\gamma_{1}\left(1-e^{u_{n}^{\delta}}\right)+\gamma_{2}\left(1-e^{v_{k}^{\delta}}\right)} \prod_{i=1}^{n} u_{i}^{\delta-1} \prod_{j=1}^{k} v_{j}^{\delta-1}
$$

and the corresponding log-likelihood function is

$$
\begin{aligned}
& L\left(\gamma_{1}, \gamma_{2}, \delta\right)=\log L_{O}\left(\gamma_{1}, \gamma_{2}, \delta ; \mathrm{u}, \mathrm{v}\right) \\
& =n \log \gamma_{1}+k \log \gamma_{2}+(n+k) \log \delta+\gamma_{1}\left(1-e^{u_{n}^{\delta}}\right)+\gamma_{2}\left(1-e^{v_{k}^{\delta}}\right) \\
& +\sum_{i=1}^{n} u_{i}^{\delta}+\sum_{j=1}^{k} v_{j}^{\delta}+(\delta-1)\left(\sum_{i=1}^{n} \log u_{i}+\sum_{j=1}^{k} \log v_{j}\right) .
\end{aligned}
$$

The ML estimate of the parameters $\gamma_{1}, \gamma_{2}$ and $\delta$, say $\hat{\gamma}_{1}, \hat{\gamma}_{2}$ and $\hat{\delta}$, can be obtained using the following system of equations:

$$
\begin{array}{r}
\frac{\partial L}{\partial \gamma_{1}}=\frac{n}{\gamma_{1}}+1-e^{u_{n}^{\delta}}=0, \\
\frac{\partial L}{\partial \gamma_{2}}=\frac{k}{\gamma_{2}}+1-e^{\nu_{k}^{\delta}}=0
\end{array}
$$

and

$$
\frac{\partial L}{\partial \delta}=\frac{n+k}{\delta}-\gamma_{1} u_{n}^{\delta} e^{u_{n}^{\delta}} \log u_{n}-\gamma_{2} v_{k}^{\delta} e^{v_{k}^{\delta}} \log v_{k}+\sum_{i=1}^{n} \log u_{i}\left(1+u_{i}^{\delta}\right)+\sum_{j=1}^{k} \log v_{j}\left(1+v_{j}^{\delta}\right)=0
$$

Then, by using the invariance property of the MLEs, the ML estimate of $R$ can be computed as

$$
\hat{R}=\sum_{i=r}^{m} \sum_{j=0}^{m-i}(-1)^{j}\left(\begin{array}{c}
m \\
i
\end{array}\right)\left(\begin{array}{c}
m-i \\
j
\end{array}\right) \frac{\hat{\gamma}_{2}}{(i+j) \hat{\gamma}_{1}+\hat{\gamma}_{2}}
$$

\section{Bayesian analyses}

In the Bayesian setting, the observer combines subjective opinion based on insight or experience with the available observations to get balanced values and to update the estimates as more information and data become accessible. The parameters of interest are assumed to be random variables with speified prior probability distributions. Then, in order to conduct a Bayesian analysis, usually quadratic loss function is considered. A very popular quadratic loss is the squared error (SE) loss function given by $L_{S E}(g(\theta), \hat{g}(\theta))=(g(\theta)-\hat{g}(\theta))^{2}$ where $\hat{g}(\theta)$ is an estimate of the parametric function $g(\theta)$. It is well known that, under this loss function, the Bayes estimate of $g(\theta)$, say $\hat{g}_{S E}(\theta)$, is the posterior mean.

Using SE loss function in the Bayesian approach leads to the equal penalization for underestimation and overestimation which is inappropriate in some practical situations. For instance, in estimating the reliability characteristics overestimation is more serious than the underestimation. Therefore, different asymmetric loss functions are considered by researchers such as Linex and general entropy (GE) loss functions for implementing Bayesian method in various field of reliability inference (see for example Ahmed (2014), Nadar and Kizilaslan (2016) and Tarvirdizade and Ahmadpour (2016)). The Linex loss function given by 


$$
L_{L E}(g(\theta), \hat{g}(\theta))=\exp [v(g(\theta)-\hat{g}(\theta))]-v(g(\theta)-\hat{g}(\theta))-1, \quad v \neq 0,
$$

is a popular asymmetric loss function that penalizes underestimation and overestimation for negative and positive $v$, respectively. Under this loss function, the Bayesian estimate of $g(\theta)$ is given by

$$
\hat{g}_{L E}(\theta)=-\frac{1}{v} \log \left(E_{\theta}[\exp (-v g(\theta)) \mid \text { data }]\right) .
$$

Another important asymmetric loss is the GE loss function defined as

$$
L_{G E}(g(\theta), \hat{g}(\theta)) \propto\left(\frac{\hat{g}(\theta)}{g(\theta)}\right)^{w}-w \log \left(\frac{\hat{g}(\theta)}{g(\theta)}\right)-1, \quad w \neq 0 .
$$

Based on this loss function, the Bayes estimate of $g(\theta)$ is given by

$$
\hat{g}_{G E}(\theta)=\left(E_{\theta}\left[(g(\theta))^{-w} \mid \text { data }\right]\right)^{-1 / w} \text {. }
$$

In this section we derive different Bayes estimates of the reliability $R$ by using the above mentioned loss functions. We assume that $\gamma_{1}, \gamma_{2}$ and $\delta$ are independent r.v.s and follow the gamma prior distributions as

$$
\left\{\begin{array}{l}
\pi_{1}\left(\gamma_{1}\right) \propto \gamma_{1}^{a_{1}-1} e^{-\gamma_{1} b_{1}} \\
\pi_{2}\left(\gamma_{2}\right) \propto \gamma_{2}^{a_{2}-1} e^{-\gamma_{2} b_{2}} \\
\pi_{3}(\delta) \propto \delta^{a_{3}-1} e^{-\delta b_{3}}
\end{array}\right.
$$

respectively, where the hyperparameters $a_{i}, b_{i}, i=1,2,3$ are positive. By combining (5) with (13), the joint density function of $\gamma_{1}, \gamma_{2}, \delta$ and the data can be written as

$$
\begin{aligned}
& \pi\left(\gamma_{1}, \gamma_{2}, \delta ; \mathrm{u}, \mathrm{v}\right) \propto \gamma_{1}^{n+a_{1}-1} \gamma_{2}^{k+a_{2}-1} \delta^{n+k+a_{3}-1} e^{\sum_{i=1}^{n} u_{i}^{\delta}+\sum_{j=1}^{k} v_{j}^{\delta}-b_{3} \delta} \\
& e^{\gamma_{1}\left(-b_{1}+1-e^{u^{\delta}}\right)+\gamma_{2}\left(-b_{2}+1-e^{\nu_{k}^{\delta}}\right)} \prod_{i=1}^{n} u_{i}^{\delta-1} \prod_{j=1}^{k} v_{j}^{\delta-1} .
\end{aligned}
$$

Thus, we can write the posterior density function of $\gamma_{1}, \gamma_{2}$ and $\delta$ as

$$
\pi^{*}\left(\gamma_{1}, \gamma_{2}, \delta \mid \mathrm{u}, \mathrm{v}\right)=\frac{1}{C} \pi\left(\gamma_{1}, \gamma_{2}, \delta ; \mathrm{u}, \mathrm{v}\right)
$$

in which

$$
C=\iint_{0}^{\infty} \int_{0}^{\infty} \int_{0}^{\infty} \pi\left(\gamma_{1}, \gamma_{2}, \delta ; \mathrm{u}, \mathrm{v}\right) d \gamma_{1} d \gamma_{2} d \delta
$$

In the following, by using the loss functions $L_{S E}, L_{L E}$ and $L_{E N}$, we obtain the Bayes estimates of reliability parameter. First, assuming squared error loss function $L_{S E}$, the estimates of $R$ becomes

$$
\hat{R}_{S E}=E(R \mid \mathrm{u}, \mathrm{v})=\frac{1}{C} \iint_{0}^{\infty} \int_{0}^{\infty} \int_{0}^{\infty} R \pi\left(\gamma_{1}, \gamma_{2}, \delta, \text { data }\right) d \gamma_{1} d \gamma_{2} d \delta .
$$

Next, against the loss $L_{L E}$, the Bayes estimate of reliability $R$ can be obtained as

$$
\hat{R}_{L E}=-\frac{1}{v} \log (E[\exp (-v R) \mid \mathrm{u}, \mathrm{v}])
$$




$$
=-\frac{1}{v} \log \frac{1}{C} \int_{0}^{\infty} \int_{0}^{\infty} \int_{0}^{\infty} \exp (-v R) \pi\left(\gamma_{1}, \gamma_{2}, \delta, \text { data }\right) d \gamma_{1} d \gamma_{2} d \delta .
$$

Similarly, considering the entropy loss function $L_{E N}$, we obtain the Bayes estimate of the parameter $R$ as

$$
\hat{R}_{E N}=E\left[R^{-w} \mid \mathrm{u}, \mathrm{v}\right]^{-\frac{1}{w}}
$$

in which the required conditional expectation is computed as

$$
E\left[R^{-w} \mid \mathrm{u}, \mathrm{v}\right]=\frac{1}{C} \int_{0}^{\infty} \iint_{0}^{\infty} \int_{0}^{\infty} R^{-w} \pi\left(\gamma_{1}, \gamma_{2}, \delta, \text { data }\right) d \gamma_{1} d \gamma_{2} d \delta
$$

It is easily seen that all the above estimators are involved the ratio of two integrals for which simplified closed forms can not be obtained. Therefore, in the following, we employ an approximation procedure, namely Lindley's approximation, to calculate the Bayes estimates.

Let $h\left(\gamma_{1}, \gamma_{2}, \delta\right)$ be any function of the parameters and consider

$$
\begin{aligned}
E\left(h\left(\gamma_{1}, \gamma_{2}, \delta\right) \mid \mathrm{u}, \mathrm{v}\right)=\int_{0}^{\infty} \int_{0}^{\infty} \int_{0}^{\infty} h\left(\gamma_{1}, \gamma_{2}, \delta\right) \pi^{*}\left(\gamma_{1}, \gamma_{2}, \delta \mid \mathrm{u}, \mathrm{v}\right) d \gamma_{1} \gamma_{2} d \delta \\
=\frac{\int_{0}^{\infty} \int_{0}^{\infty} \int_{0}^{\infty} h\left(\gamma_{1}, \gamma_{2}, \delta\right)\left\{\exp \left(L\left(\gamma_{1}, \gamma_{2}, \delta ; \mathrm{u}, \mathrm{v}\right)+\eta\left(\gamma_{1}, \gamma_{2}, \delta\right)\right)\right\} d \gamma_{1} \gamma_{2} d \delta}{\int_{0}^{\infty} \int_{0}^{\infty} \int_{0}^{\infty} \exp \left(L\left(\gamma_{1}, \gamma_{2}, \delta ; \mathrm{u}, \mathrm{v}\right)+\eta\left(\gamma_{1}, \gamma_{2}, \delta\right)\right) d \gamma_{1} \gamma_{2} d \delta} .
\end{aligned}
$$

in which $\eta\left(\gamma_{1}, \gamma_{2}, \delta\right)=\log \left(\pi_{1}\left(\gamma_{1}\right) \pi_{2}\left(\gamma_{2}\right) \pi_{3}(\delta)\right)$ and $L\left(\gamma_{1}, \gamma_{2}, \delta ; \mathrm{u}, \mathrm{v}\right)$ is the log-likelihood function of the parameters given by expression (6). Applying Lindley's procedure (1980), $E\left(h\left(\gamma_{1}, \gamma_{2}, \delta\right) \mid \mathrm{u}, \mathrm{v}\right)$ are approximated by

$$
\begin{aligned}
& h\left(\gamma_{1}, \gamma_{2}, \delta\right)+\frac{1}{2} \sum h_{i j} \tau_{i j}+\sum V_{j} \eta_{j}+\frac{1}{2} \sum L_{i i i} \tau_{i i} V_{i}+\frac{1}{2} L_{112}\left(2 \tau_{122} V_{1}+\tau_{11} V_{2}\right) \\
& +\frac{1}{2}\left\{L_{122}\left(\tau_{22} V_{1}+2 \tau_{12} V_{2}\right)+L_{133}\left(\tau_{33} V_{1}+2 \tau_{13} V_{3}\right)+L_{223}\left(\tau_{22} V_{3}+2 \tau_{23} V_{2}\right)\right\} \\
& +\frac{1}{2}\left\{L_{233}\left(\tau_{33} V_{2}+2 \tau_{23} V_{3}\right)+L_{123}\left(2 \tau_{23} V_{1}+2 \tau_{12} V_{3}\right)+L_{113}\left(\tau_{11} V_{3}+2 \tau_{13} V_{1}\right)\right\}
\end{aligned}
$$

where $V_{r}=\sum_{j} h_{j} \tau_{r j}$ with $\tau_{i j}$ being the $(i, j)$ th elements of the matrix $\left[-\partial^{2} L / \partial \gamma_{i} \partial \gamma_{j}\right]^{-1}$ and each suffix of variables $h, \eta$ or $L$ denotes differentiation once with respect to the variable having that suffix. Calculating all the expressions in (21) at the ML estimates $\hat{\gamma}_{1}$, $\hat{\gamma}_{2}$ and $\hat{\delta}$, the approximate Bayes estimate of $R$ is obtained. Several have used the above approximation in situations where the explicit forms of Bayes estimators are unavailable; see for example Nadar and Kizilaslan (2016), Tarvirdizade and Ahmadpour (2016) and Ahmed (2014).

In our case, the required quantities in expression (21) are derived as 


$$
\begin{gathered}
L_{11}=-\frac{n}{\gamma_{1}^{2}}, \quad L_{22}=-\frac{k}{\gamma_{2}^{2}}, \quad L_{12}=L_{21}=0, \\
L_{13}=L_{31}=-u_{n}^{\delta} e^{u_{n}^{\delta}} \log u_{n}, \quad L_{23}=L_{32}=-v_{k}^{\delta} e^{v_{k}^{\delta}} \log v_{k}, \\
L_{33}=\frac{\partial^{2} L}{\partial \delta^{2}}=-\frac{n+k}{\delta^{2}}+\sum_{i=1}^{n}\left(\log u_{i}\right)^{2} u_{i}^{\delta}+\sum_{j=1}^{k}\left(\log v_{j}\right)^{2} v_{j}^{\delta} \\
-\gamma_{1} u_{n}^{\delta}\left(\log u_{n}\right)^{2} e^{u_{n}^{\delta}}\left(1+u_{n}^{\delta}\right)-\gamma_{2} v_{k}^{\delta}\left(\log v_{k}\right)^{2} e^{v_{k}^{\delta}}\left(1+v_{k}^{\delta}\right), \\
L_{112}=L_{113}=L_{221}=L_{223}=L_{121}=L_{211}=L_{122}=L_{123}=L_{231}=L_{132}=0, \\
L_{111}=\frac{2 n}{\gamma_{1}^{3}}, \quad L_{133}=L_{331}=-u_{n}^{\delta}\left(\log u_{n}\right)^{2} e^{u_{n}^{\delta}}\left(1+u_{n}^{\delta}\right), \\
L_{222}=\frac{2 k}{\gamma_{2}^{3}}, \quad L_{233}=L_{332}=-v_{k}^{\delta}\left(\log v_{k}\right)^{2} e^{v_{k}^{\delta}}\left(1+v_{k}^{\delta}\right), \\
L_{333}=\frac{2(n+k)}{\delta^{3}}+\sum_{i=1}^{n} u_{i}^{\delta}\left(\log u_{i}\right)^{3}+\sum_{j=1}^{k} v_{j}^{\delta}\left(\log v_{j}\right)^{3} \\
-\gamma_{1} \sum_{i=1}^{n} u_{n}^{\delta}\left(\log u_{n}\right)^{3} e^{u_{n}^{\delta}}\left(1+3 u_{n}^{\delta}+u_{n}^{2 \delta}\right)-\gamma_{2} \sum_{j=1}^{k} v_{k}^{\delta}\left(\log v_{k}\right)^{3} e^{v_{k}^{\delta}}\left(1+3 v_{k}^{\delta}+v_{k}^{2 \delta}\right), \\
\eta_{1}=\frac{a_{1}-1}{\gamma_{1}}-b_{1}, \eta_{2}=\frac{a_{2}-1}{\gamma_{2}}-b_{2}, \eta_{3}=\frac{a_{3}-1}{\delta}-b_{3} .
\end{gathered}
$$

Now, for computing the approximate Bayes estimate of $R$ based on SE loss function, let $h\left(\gamma_{1}, \gamma_{2}, \delta\right)=R$. Hence, $h_{3}=h_{13}=h_{31}=h_{23}=h_{32}=h_{33}=0$ and we have

$$
\begin{aligned}
& \hat{R}_{S E} \approx \hat{R}+\frac{1}{2}\left(R_{11} \tau_{11}+R_{22} \tau_{22}+2 R_{12} \tau_{12}\right)+V_{1} \eta_{1}+V_{2} \eta_{2}+V_{3} \eta_{3} \\
& +\frac{1}{2}\left(L_{111} \tau_{11} V_{1}+L_{222} \tau_{22} V_{2}+L_{333} \tau_{33} V_{3}\right)+\frac{1}{2} L_{133}\left(\tau_{33} V_{1}+2 \tau_{13} V_{3}\right) \\
& +\frac{1}{2} L_{233}\left(\tau_{33} V_{2}+2 \tau_{23} V_{3}\right)
\end{aligned}
$$

where $V_{1}=R_{1} \tau_{11}+R_{2} \tau_{12}, V_{2}=R_{1} \tau_{21}+R_{2} \tau_{22}, V_{3}=R_{1} \tau_{31}+R_{2} \tau_{32}$ and

$$
\begin{gathered}
R_{1}=\frac{\partial R}{\partial \gamma_{1}}=-\sum_{i=r}^{m} \sum_{j=0}^{m-i}(-1)^{j}\left(\begin{array}{c}
m \\
i
\end{array}\right)\left(\begin{array}{c}
m-i \\
j
\end{array}\right) \frac{\gamma_{2}(i+j)}{\left((i+j) \gamma_{1}+\gamma_{2}\right)^{2}}, \\
R_{2}=\frac{\partial R}{\partial \gamma_{2}}=\sum_{i=r}^{m} \sum_{j=0}^{m-i}(-1)^{j}\left(\begin{array}{c}
m \\
i
\end{array}\right)\left(\begin{array}{c}
m-i \\
j
\end{array}\right) \frac{\gamma_{1}(i+j)}{\left((i+j) \gamma_{1}+\gamma_{2}\right)^{2}}, \\
R_{11}=\frac{\partial^{2} R}{\partial \gamma_{1}^{2}}=+\sum_{i=r}^{m} \sum_{j=0}^{m-i}(-1)^{j}\left(\begin{array}{c}
m \\
i
\end{array}\right)\left(\begin{array}{c}
m-i \\
j
\end{array}\right) \frac{2 \gamma_{2}(i+j)^{2}}{\left((i+j) \gamma_{1}+\gamma_{2}\right)^{3}}, \\
R_{22}=\frac{\partial^{2} R}{\partial \gamma_{2}^{2}}=-\sum_{i=r}^{m} \sum_{j=0}^{m-i}(-1)^{j}\left(\begin{array}{c}
m \\
i
\end{array}\right)\left(\begin{array}{c}
m-i \\
j
\end{array}\right) \frac{2 \gamma_{1}(i+j)}{\left((i+j) \gamma_{1}+\gamma_{2}\right)^{3}},
\end{gathered}
$$




$$
\begin{aligned}
& R_{12}=\frac{\partial^{2} R}{\partial \gamma_{1} \partial \gamma_{2}} \\
& =\sum_{i=r}^{m} \sum_{j=0}^{m-i}(-1)^{j}\left(\begin{array}{c}
m \\
i
\end{array}\right)\left(\begin{array}{c}
m-i \\
j
\end{array}\right)\left\{-\frac{i+j}{\left((i+j) \gamma_{1}+\gamma_{2}\right)^{2}}+\frac{2 \gamma_{2}(i+j)}{\left((i+j) \gamma_{1}+\gamma_{2}\right)^{3}}\right\} .
\end{aligned}
$$

Next, under LE loss function, the approximate Bayes estimate of reliability is obtained by choosing $h\left(\gamma_{1}, \gamma_{2}, \delta\right)=e^{-v R}$. Then, it is easily seen that $h_{1}=-v e^{-v R} R_{1}$, $h_{2}=-v e^{-g R} R_{2}, h_{11}=v e^{-v R}\left[v R_{1}^{2}-R_{11}\right], h_{22}=v e^{-v R}\left[v R_{2}^{2}-R_{22}\right]$ and $h_{12}=v e^{-v R}\left[v R_{1} R_{2}-R_{12}\right]$ where $R_{1}, R_{2}, R_{11}, R_{22}, R_{12}$ are given by (23)-(27), respectively. So, we have

$$
\hat{R}_{L E} \approx-\frac{1}{v} \log \left\{e^{-v \hat{R}}+S^{*}\right\}
$$

with

$$
\begin{aligned}
& S^{*}=\frac{1}{2}\left(h_{11} \tau_{11}+h_{22} \tau_{22}+2 h_{12} \tau_{12}\right)+V_{1} \eta_{1}+V_{2} \eta_{2}+V_{3} \eta_{3} \\
& +\frac{1}{2}\left(L_{111} \tau_{11} V_{1}+L_{222} \tau_{22} V_{2}+L_{333} \tau_{33} V_{3}\right)+\frac{1}{2} L_{133}\left(\tau_{33} V_{1}+2 \tau_{13} V_{3}\right) \\
& +\frac{1}{2} L_{233}\left(\tau_{33} V_{2}+2 \tau_{23} V_{3}\right)
\end{aligned}
$$

Finally, considering $h\left(\gamma_{1}, \gamma_{2}, \delta\right)=R^{-w}$, the approximate Bayes estimate of reliability under GE loss function is derived as

$$
\hat{R}_{G E} \approx\left\{R^{-w}+S^{*}\right\}^{-\frac{1}{w}}
$$

where $S^{*}$ is given by (29) and

$$
\begin{aligned}
& h_{1}=-w R^{-w-1} R_{1}, h_{2}=-w R^{-w-1} R_{2}, h_{11}=w(w+1) R^{-w-2} R_{1}^{2}-w R^{-w-1} R_{11}, \\
& h_{22}=w(w+1) R^{-w-2} R_{2}^{2}-w R^{-w-1} R_{22} \text { and } h_{12}=w(w+1)^{-w-2} R_{1} R_{2}-w R^{-w-1} R_{12} .
\end{aligned}
$$

\section{Numerical Comparisons}

\subsection{Simulation study}

In order to investigate the behaviour of the different methods, Monte Carlo simulations are performed in this section. The performances of the classical and Bayesian estimates of the reliability parameter are compared based on their mean squared errors (MSE) and average values (AV). R 2.14.0 is used for all the computation of the different procedures.

We have considered two sets of parameter values as $\left(\gamma_{1}, \gamma_{2}, \delta\right)=(3,1,0.5),(2,4,0.5)$ and different choices of sample sizes as $(n, k)=(5,5),(8,8),(10,10),(15,15)$. With these choices of the parameter values, the true value of reliability $R_{r, m}$ for $(r, m)=(1,3)$ becomes, respectively, 0.421428 and 0.9 and for $(r, m)=(2,4)$ becomes 0.287912 and 0.8 . In each case, different random samples are generated from BSH model and the ML estimates of the unknown parameters $\gamma_{1}, \gamma_{2}$ and $\delta$ are obtained from the system of equations in (7)-(9). Then, the ML estimates of the 
reliability $R_{r, m}$ are computed from relation (10). The AVs and MSEs of the MLEs obtained from 10000 replications are presented in Table 1.

To evaluate the Bayes estimates, we take two different sets of hyper-parameter values as

Prior I: $a_{i}=1, b_{i}=2, i=1,2,3$ and Prior II: $a_{i}=3, b_{i}=1, i=1,2,3$.

For the above cases, the approximate Bayes estimates of the reliability parameter against squared error, linex and entropy loss functions are computed using Lindley approximation. To this end, we have considered three different choices of $v$ and $w$ as $-0.5,1,1.5$ for both linex and entropy loss functions. Tables $2-5$ present the AVs and MSEs of the estimates obtained from 10000 replications. The following points are observed from the tabulated values.

(1) The ML estimates obtained based on larger sample size $n$ have smaller MSEs as we expected. Similar improvements are observed for the Bayes estimates evaluated from different loss functions.

(2) The Bayes estimates of the reliability $R$ based on SE, linex and GE loss functions give better performances than the MLEs in terms of minimum MSEs. The choice $v=1.5$ for the linex loss seems to be reasonable while for the GE loss function, $w=-0.5$ provides better performances. Moreover, among different Bayes estimators of $R, \hat{R}_{L E}$ with $v=1.5$ is superior than their respective competitors.

Table 1: ML estimate of the reliability $R_{r, m}$ for various sample sizes.

\begin{tabular}{|c|c|c|c|c|c|}
\hline \multirow{2}{*}{$(r, m)$} & \multirow{2}{*}{$(n, k)$} & \multicolumn{2}{|c|}{$\left(\gamma_{1}, \gamma_{2}, \delta\right)=(3,1,0.5)$} & \multicolumn{2}{c|}{$\left(\gamma_{1}, \gamma_{2}, \delta\right)=(2,4,0.5)$} \\
\cline { 3 - 6 } & & \multicolumn{2}{|c|}{$\hat{R}$} & \multicolumn{2}{c|}{$\hat{R}$} \\
\hline \multirow{3}{*}{$(1,3)$} & & $\mathrm{AV}$ & $\mathrm{MSE}$ & $\mathrm{AV}$ & $\mathrm{MSE}$ \\
\cline { 2 - 6 } & $(5,5)$ & 0.293642 & 0.032005 & 0.934008 & 0.0149181 \\
\cline { 2 - 6 } & $(8,8)$ & 0.339948 & 0.026195 & 0.923687 & 0.005268 \\
\cline { 2 - 6 } & $(10,10)$ & 0.353079 & 0.020056 & 0.920232 & 0.002911 \\
\cline { 2 - 6 } & $(15,15)$ & 0.377698 & 0.012042 & 0.913427 & 0.002278 \\
\hline \multirow{3}{*}{$(2,4)$} & $(5,5)$ & 0.198313 & 0.021789 & 0.864787 & 0.017536 \\
\cline { 2 - 6 } & $(8,8)$ & 0.226007 & 0.014046 & 0.844653 & 0.008193 \\
\cline { 2 - 6 } & $(10,10)$ & 0.239544 & 0.011037 & 0.837603 & 0.005903 \\
\cline { 2 - 6 } & $(15,15)$ & 0.256809 & 0.006831 & 0.824692 & 0.003709 \\
\hline
\end{tabular}

Table 2: Avs and MSEs of the Bayes estimates of the reliability $R_{r, m}$ based on prior I when $\left(\gamma_{1}, \gamma_{2}, \delta\right)=(3,1,0.5)$.

\begin{tabular}{|c|c|c|c|c|c|c|c|c|}
\hline$(r, m)$ & $(n, k)$ & $\hat{R}_{S E}$ & \multicolumn{3}{|c|}{$\hat{R}_{L E}$} & \multicolumn{3}{c|}{$\hat{R}_{G E}$} \\
\hline & & & $v=-0.5$ & $v=1$ & $v=1.5$ & $w=-0.5$ & $v=1$ & $v=1.5$ \\
\hline \multirow{3}{*}{$(1,3)$} & \multirow{2}{*}{$5,5)$} & 0.483273 & 0.491411 & 0.467279 & 0.459231 & 0.463877 & 0.468145 & 0.494912 \\
\cline { 3 - 9 } & & 0.017259 & 0.018275 & 0.015548 & 0.014821 & 0.016439 & 0.019653 & 0.023246 \\
\cline { 3 - 9 } & \multirow{2}{*}{$(8,8)$} & 0.429573 & 0.435377 & 0.418220 & 0.412680 & 0.411148 & 0.411925 & 0.414721 \\
\cline { 3 - 9 } & & 0.012687 & 0.012874 & 0.012450 & 0.012437 & 0.013310 & 0.014891 & 0.015227 \\
\cline { 2 - 8 } & \multirow{2}{*}{$(10,10)$} & 0.415727 & 0.420572 & 0.406244 & 0.401619 & 0.402768 & 0.403171 & 0.405270 \\
\hline
\end{tabular}




\begin{tabular}{|c|c|c|c|c|c|c|c|c|}
\hline & & 0.010788 & 0.010897 & 0.010817 & 0.010761 & 0.011510 & 0.012841 & 0.013450 \\
\hline & \multirow[t]{2}{*}{$(15,15)$} & 0.401261 & 0.404653 & 0.394599 & 0.391329 & 0.355239 & 0.364273 & 0.392407 \\
\hline & & 0.007903 & 0.008257 & 0.008122 & 0.007820 & 0.008344 & 0.008559 & 0.008736 \\
\hline \multirow[t]{8}{*}{$(2,4)$} & \multirow[t]{2}{*}{$(5,5)$} & 0.318644 & 0.325357 & 0.315817 & 0.308699 & 0.317020 & 0.321571 & 0.323262 \\
\hline & & 0.014731 & 0.015884 & 0.012736 & 0.011883 & 0.011935 & 0.012814 & 0.013024 \\
\hline & \multirow[t]{2}{*}{$(8,8)$} & 0.308099 & 0.312399 & 0.299853 & 0.295896 & 0.293941 & 0.294875 & 0.298119 \\
\hline & & 0.008812 & 0.009153 & 0.008248 & 0.008019 & 0.008618 & 0.009072 & 0.009225 \\
\hline & \multirow{2}{*}{$(10,10)$} & 0.294431 & 0.297899 & 0.287751 & 0.284582 & 0.281197 & 0.282605 & 0.284724 \\
\hline & & 0.007046 & 0.007217 & 0.006776 & 0.006675 & 0.007131 & 0.007284 & 0.007335 \\
\hline & \multirow[t]{2}{*}{$(15,15)$} & 0.279314 & 0.281599 & 0.274867 & 0.272704 & 0.267782 & 0.270343 & 0.271193 \\
\hline & & 0.004767 & 0.004792 & 0.004674 & 0.004631 & 0.005006 & 0.005227 & 0.005314 \\
\hline
\end{tabular}

Table 3: Avs and MSEs of the Bayes estimates of the reliability $R_{r, m}$ based on prior I when $\left(\gamma_{1}, \gamma_{2}, \delta\right)=(2,4,0.5)$.

\begin{tabular}{|c|c|c|c|c|c|c|c|c|}
\hline$(r, m)$ & $(n, k)$ & $\hat{R}_{S E}$ & \multicolumn{3}{|c|}{$\hat{R}_{L E}$} & \multicolumn{3}{|c|}{$\hat{R}_{G E}$} \\
\hline & & & $v=-0.5$ & $v=1$ & $v=1.5$ & $w=-0.5$ & $v=1$ & $v=1.5$ \\
\hline \multirow[t]{8}{*}{$(1,3)$} & \multirow[t]{2}{*}{$(5,5)$} & 0.825742 & 0.829608 & 0.817510 & 0.813109 & 0.820296 & 0.833427 & 0.837241 \\
\hline & & 0.008816 & 0.009345 & 0.007861 & 0.007227 & 0.010176 & 0.011855 & 0.012976 \\
\hline & \multirow[t]{2}{*}{$(8,8)$} & 0.862979 & 0.865149 & 0.858402 & 0.855957 & 0.860149 & 0.870506 & 0.873205 \\
\hline & & 0.003492 & 0.004256 & 0.003981 & 0.003275 & 0.003801 & 0.004178 & 0.004496 \\
\hline & \multirow[t]{2}{*}{$(10,10)$} & 0.877564 & 0.879183 & 0.874172 & 0.872394 & 0.875522 & 0.881733 & 0.882219 \\
\hline & & 0.002328 & 0.002735 & 0.002589 & 0.002212 & 0.002488 & 0.002695 & 0.002782 \\
\hline & \multirow[t]{2}{*}{$(15,15)$} & 0.895825 & 0.896781 & 0.893745 & 0.892422 & 0.894678 & 0.896993 & 0.897607 \\
\hline & & 0.001198 & 0.001304 & 0.001236 & 0.001169 & 0.001239 & 0.001391 & 0.001453 \\
\hline \multirow[t]{8}{*}{$(2,4)$} & \multirow[t]{2}{*}{$(5,5)$} & 0.716446 & 0.722540 & 0.703742 & 0.0 .697141 & 0.706634 & 0.718588 & 0.726193 \\
\hline & & 0.011236 & 0.011527 & 0.011091 & 0.010422 & 0.010491 & 0.011822 & 0.013196 \\
\hline & \multirow[t]{2}{*}{$(8,8)$} & 0.762071 & 0.766073 & 0.753894 & 0.749629 & 0.756235 & 0.757033 & 0.757791 \\
\hline & & 0.006046 & 0.007427 & 0.006920 & 0.005668 & 0.006709 & 0.007365 & 0.007421 \\
\hline & \multirow[t]{2}{*}{$(10,10)$} & 0.780721 & 0.783855 & 0.774218 & 0.770850 & 0.776259 & 0.779630 & 0.780197 \\
\hline & & 0.004538 & 0.005258 & 0.004993 & 0.004347 & 0.004881 & 0.005324 & 0.005580 \\
\hline & \multirow[t]{2}{*}{$(15,15)$} & 0.803978 & 0.806053 & 0.799724 & 0.797543 & 0.801198 & 0.803917 & 0.804235 \\
\hline & & 0.002896 & 0.003023 & 0.002931 & 0.002871 & 0.002959 & 0.003273 & 0.003428 \\
\hline
\end{tabular}

Table 4: Avs and MSEs of the Bayes estimates of the reliability $R_{r, m}$ based on prior II when $\left(\gamma_{1}, \gamma_{2}, \delta\right)=(3,1,0.5)$.

\begin{tabular}{|c|c|c|c|c|c|c|c|c|}
\hline$(r, m)$ & $(n, k)$ & $\hat{R}_{S E}$ & \multicolumn{3}{|c|}{$\hat{R}_{L E}$} & \multicolumn{3}{|c|}{$\hat{R}_{G E}$} \\
\hline & & & $v=-0.5$ & $v=1$ & $v=1.5$ & $w=-0.5$ & $w=1$ & $w=1.5$ \\
\hline \multirow[t]{7}{*}{$(1,3)$} & \multirow[t]{2}{*}{$(5,5)$} & 0.459806 & 0.466306 & 0.447041 & 0.440802 & 0.444319 & 0.461559 & 0.475058 \\
\hline & & 0.008998 & \begin{tabular}{|l|}
0.009516 \\
\end{tabular} & 0.008191 & 0.007897 & 0.008534 & 0.010855 & 0.013167 \\
\hline & \multirow[t]{2}{*}{$(8,8)$} & 0.453824 & 0.458068 & 0.445372 & 0.441360 & \begin{tabular}{|l|}
0.443907 \\
\end{tabular} & 0.465036 & 0.473550 \\
\hline & & 0.006925 & 0.007219 & 0.006440 & 0.006235 & 0.006631 & 0.007051 & 0.007714 \\
\hline & \multirow[t]{2}{*}{$(10,10)$} & 0.456322 & 0.460738 & 0.448129 & 0.441007 & 0.446623 & 0.469503 & 0.473036 \\
\hline & & 0.006438 & 0.006853 & 0.006409 & 0.006193 & 0.006588 & 0.006813 & 0.007426 \\
\hline & $(15,15)$ & 0.451069 & \begin{tabular}{|l|l}
0.453173 \\
\end{tabular} & 0.443920 & 0.440897 & 0.442850 & 0.447931 & 0.451852 \\
\hline
\end{tabular}


Bayesian inference on reliability in a multicomponent stress-strength bathtub-shaped model based on record values

\begin{tabular}{|c|c|c|c|c|c|c|c|c|}
\hline & & 0.005672 & 0.005863 & 0.005346 & 0.005210 & 0.005439 & 0.005478 & 0.005735 \\
\hline \multirow{8}{*}{$(2,4)$} & \multirow{2}{*}{$(5,5)$} & 0.332754 & 0.337762 & 0.323151 & 0.318552 & 0.317432 & 0.346150 & 0.352719 \\
\hline & & 0.007351 & 0.007903 & 0.006421 & 0.006034 & 0.006431 & 0.006985 & 0.007612 \\
\hline & \multirow[t]{2}{*}{$(8,8)$} & 0.323085 & 0.326199 & 0.317086 & 0.314099 & 0.313372 & 0.332491 & 0.378116 \\
\hline & & 0.005271 & 0.005547 & 0.004787 & 0.004621 & 0.004857 & 0.004934 & 0.005762 \\
\hline & \multirow[t]{2}{*}{$(10,10)$} & 0.325283 & 0.328417 & 0.319195 & 0.316240 & 0.315577 & 0.318205 & 0.331719 \\
\hline & & 0.005336 & 0.005625 & 0.004826 & 0.004503 & 0.004647 & 0.004981 & 0.005183 \\
\hline & \multirow[t]{2}{*}{$(15,15)$} & 0.317691 & 0.319914 & 0.313579 & 0.311205 & 0.310662 & 0.316567 & 0.321297 \\
\hline & & 0.004175 & 0.004356 & 0.003885 & 0.003741 & 0.003855 & 0.004371 & 0.004462 \\
\hline
\end{tabular}

Table 5: Avs and MSEs of the Bayes estimates of the reliability $R_{r, m}$ based on prior II when $\left(\gamma_{1}, \gamma_{2}, \delta\right)=(2,4,0.5)$.

\begin{tabular}{|c|c|c|c|c|c|c|c|c|}
\hline$(r, m)$ & $(n, k)$ & $\hat{R}_{S E}$ & \multicolumn{3}{|c|}{$\hat{R}_{L E}$} & \multicolumn{3}{c|}{$\hat{R}_{G E}$} \\
\hline & & & $v=-0.5$ & $v=1$ & $v=1.5$ & $w=-0.5$ & $w=1$ & $w=1.5$ \\
\hline$(1,3)$ & $(5,5)$ & 0.863276 & 0.865451 & 0.858244 & 0.855573 & 0.860148 & 0.864369 & 0.867108 \\
\hline & & 0.003216 & 0.004028 & 0.003733 & 0.002990 & 0.003542 & 0.004836 & 0.005408 \\
\hline & $(8,8)$ & 0.879105 & 0.880726 & 0.875766 & 0.874014 & 0.877112 & 0.886479 & 0.887916 \\
\hline & & 0.001815 & 0.002057 & 0.001933 & 0.001676 & 0.001848 & 0.002353 & 0.002565 \\
\hline & $(10,10)$ & 0.8818705 & 0.883034 & 0.878925 & 0.877492 & 0.880067 & 0.889157 & 0.890622 \\
\hline & & 0.001440 & 0.001689 & 0.001408 & 0.001368 & 0.001536 & 0.001893 & 0.002039 \\
\hline & $(15,15)$ & 0.887041 & 0.887962 & 0.885146 & 0.884561 & 0.885937 & 0.887240 & 0.887932 \\
\hline & & 0.001066 & 0.001139 & 0.001094 & 0.001012 & 0.001067 & 0.001134 & 0.001202 \\
\hline$(2,4)$ & $(5,5)$ & 0.764241 & 0.767528 & 0.754290 & 0.749622 & 0.756880 & 0.769067 & 0.771215 \\
\hline & & 0.005341 & 0.006763 & 0.006239 & 0.004962 & 0.006021 & 0.009018 & 0.010452 \\
\hline & $(8,8)$ & 0.781913 & 0.785510 & 0.775439 & 0.772026 & 0.777483 & 0.781044 & 0.783216 \\
\hline & & 0.003221 & 0.003833 & 0.003604 & 0.003065 & 0.003502 & 0.004732 & 0.005307 \\
\hline & $(10,10)$ & 0.783870 & 0.786544 & 0.778325 & 0.775462 & 0.780140 & 784160 & 0.785535 \\
\hline & & 0.002795 & 0.003261 & 0.003088 & 0.002674 & 0.003007 & 0.003291 & 0.003312 \\
\hline & $(15,15)$ & 0.788651 & 0.790816 & 0.784852 & 0.782801 & 0.786213 & 0.792511 & 0.793365 \\
\hline & & 0.002104 & 0.002351 & 0.002260 & 0.002039 & 0.002215 & 0.002668 & 0.002771 \\
\hline
\end{tabular}

\subsection{Data Analysis}

To understand how the proposed methods can be used in practice, let us consider the two data sets that were discussed by Tarvirdizade and Ahmadpour (2016) for BSH reliability analysis. They checked the validity of the model for data sets 1 and 2 and showed that BSH distribution fits both data sets very well. Their results about stress-strength reliability can be derived from $R_{r, m}$ by setting $m=1$. For easy reference, the data sets are presented in Tables 6 and 7.

Table 6: Data set 1

\begin{tabular}{|c|c|c|c|c|c|c|c|c|c|}
\hline 8.18 & 4.85 & 18.79 & 8.38 & 7.93 & 13.68 & 20.44 & 22.00 & 16.58 & 27.47 \\
\hline .74 & 12.32 & 7.17 & 21.26 & 14.92 & 14.35 & 7.21 & 12.30 & 33.44 & 19.67 \\
\hline .98 & 8.96 & 10.71 & 31.28 & 10.43 & \multicolumn{1}{|c|}{} \\
\cline { 1 - 7 } &
\end{tabular}

Table 7: Data set 2

\begin{tabular}{|l|l|l|l|l|l|l|l|l|l|}
\hline 12.82 & 17.86 & 7.66 & 2.48 & 8.08 & 7.35 & 11.99 & 21.00 & 7.36 & 8.11 \\
\hline
\end{tabular}




\begin{tabular}{|l|c|c|c|c|c|c|c|c|c|}
\hline .35 & 12.44 & 12.40 & 31.01 & 9.09 & 11.57 & 17.94 & 4.42 & 16.42 & 9.25 \\
\hline .96 & 13.19 & 3.21 & 13.53 & 9.08 & \multicolumn{1}{|c|}{} \\
\hline
\end{tabular}

Here, assuming three different choices of $(r, m)$ for the MSS system, we compute the estimates of reliability parameter $R_{r, m}$ by using ML and Bayesian procedures developed in this paper. From Tables 6-7, the upper record values are obtained as follows:

$$
\begin{aligned}
& \mathrm{u}=(8.18,18.79,20.44,22.00,27.47,33.44), \\
& \mathrm{v}=(12.82,17.86,21.00,24.35,31.01,37.96) .
\end{aligned}
$$

Using the above record values, the ML estimates of the parameters are computed as $\hat{\gamma}_{1}=0.050539, \hat{\gamma}_{2}=0.038180$ and $\hat{\delta}=0.446047$. So, the MLEs of the reliability $R_{r, m}$ for $(r, m)=(1,3),(2,4),(3,5)$ become $0.699700,0.512286$ and 0.416261 , respectively.

To analyze the data from the Bayesian perspective, three different sets of values for the hyper-parameters are considered as $a_{i}=b_{i}=0.0001$ (prior I), $a_{i}=2, b_{i}=3$ (prior II) and $a_{i}=4, b_{i}=2$ (prior III). Table 8 reports different Bayes estimates of $R_{r, m}$ obtained from squared error, linex and entropy loss functions. It is observed that the Bayes estimates obtained based on prior I are close to the MLEs while employing prior II and III leads to estimates that are greater than the MLEs in most of the cases.

Table 8: Bayes estimates of $R_{r, m}$ against various loss functions.

\begin{tabular}{|c|c|c|c|c|c|c|c|c|}
\hline$(r, m)$ & Prior & $\hat{R}_{S E}$ & \multicolumn{3}{|c|}{$\hat{R}_{L E}$} & \multicolumn{3}{c|}{$\hat{R}_{G E}$} \\
\hline & & & $v=-0.5$ & $v=1$ & $v=1.5$ & $w=-0.5$ & $w=1$ & $w=1.5$ \\
\hline$(1,3)$ & I & 0.663362 & 0.669881 & 0.649958 & 0.643027 & 0.652404 & 0.640443 & 0.626814 \\
\hline & II & 0.669456 & 0.703876 & 0.690546 & 0.686022 & 0.692812 & 0.670951 & 0.663072 \\
\hline & III & 0.709589 & 0.713609 & 0.701370 & 0.697157 & 0.703498 & 0.689070 & 0.675424 \\
\hline$(2,4)$ & I & 0.523565 & 0.530741 & 0.509322 & 0.502287 & 0.508991 & 0.502493 & 0.497842 \\
\hline & II & 0.557182 & 0.562459 & 0.546710 & 0.541528 & 0.547363 & 0.536039 & 0.531117 \\
\hline & III & 0.567287 & 0.572155 & 0.557329 & 0.552669 & 0.558250 & 0.541683 & 0.539918 \\
\hline$(3,5)$ & I & 0.435973 & 0.442589 & 0.423094 & .416848 & 0.4200263 & 0.411514 & 0.409686 \\
\hline & II & 0.465745 & 0.780801 & 0.455827 & 0.451064 & 0.454778 & 0.420676 & 0.417136 \\
\hline & III & 0.474991 & 0.479696 & 0.465748 & 0.461216 & 0.464856 & 0.455481 & 0.452007 \\
\hline
\end{tabular}

\section{Conclusions}

In this paper, by using upper record values, we have discussed on Bayesian estimation of stress-stregth reliability in multicomponent stress-strength bathtub-shaped model. Considering squared error, linex and general entropy loss functions, all the Bayes estimates were computed by assuming gamma priors on the parameters. Since the Bayes estimates of the interested reliability parameter could not be obtained analytically, we have provided Lindley's approach to calculate the approximate Bayes estimates. Moreover, for comparison, the maximum likelihood estimate of the reliability is derived. In order to assess the accuracy of the different estimators, Monte Carlo simulations are conducted. It is observed that the performances of the Bayes estimators based on different loss functions are superior than the corresponding ML estimators. The choice $v=1.5$ for the Linex loss function and $w=-0.5$ for the GE loss function provide Bayes estimators 
with minimum mean squared error. Moreover, we found that informative priors (prior II and III) have better performaces compared to the non-informative prior I. From Tables 15 , it is observed that by increasing sample size $n$, expected improvements are observed in the performances of all estimators.

\section{Acknowledgement}

The authors would like to thank Editor and the referees for their constructive comments and suggestions which improved and enriched the presentation of the paper.

\section{References}

1. Abd Elfattah, A.M. and Marwa O. M., (2011). Exponential distribution as a stressstrength model with type-I censored data from a Bayesian viewpoint. Pakistan Journal of Statistics and Operation Research, 7(2), 169-182.

2. Ahmed, E.A., (2014). Bayesian estimation based on progressive type II censoring from two-parameter Bathtub-shaped lifetime model: An Markov chain Monte Carlo approach. Journal of Applied Statistics, 41(4), 752-768.

3. Akgul, F.G. and Senoglu, B., (2017). Estimation of $\mathrm{P}(\mathrm{X}>\mathrm{Y})$ using some modifications of ranked set sampling for Weibull distribution. Pakistan Journal of Statistics and Operation Research, 13(4), 931-958.

4. Arnold, B.C., Balakrishnan, N., Nagaraja, H.N., (1998). Records, Vol. 768. John Wiley \& Sons.

5. Asgharzadeh, A., Fallah, A. Raqab, M.Z. and Valiollahi, R., (2018). Statistical inference based on Lindley record data. Statistical Papers, 59(2), 759-779.

6. Balakrishnan, N. and Lai, C.D., (2009). Continuous Bivariate Distributions. 2nd. Springer, New York.

7. Chen, Z. (2000). A new two-parameter lifetime distribution with bathtub shape or increasing failure rate function. Statistics and Probability Letters, 49, 155-161.

8. Dasylva, A., (2018). Design-Based Estimation with Record-Linked Administrative Files and a Clerical Review Sample. Journal of Official Statistics, 34(1), 41-54.

9. Dey, S., Mazucheli, J. and Anis, M.Z., (2016), Estimation of reliability of multicomponent stress-strength for a kumaraswamy distribution. Communications in Statistics-Theory and Methods, DOI: 10.1080/03610926.2015.1022457.

10. Hanagal, D.D., (1997). Note on estimation of reliability under bivariate Pareto stressstrength

11. model. Statistical Papers, 38, 453-459.

12. Kizilaslan, F., Nadar, M., (2015). Classical and Bayesian estimation of reliability in multicomponent stress-strength model based on Weibull distribution. Revista Colombiana de Estadistica, 38(2), 467-484.

13. Kotz, S., Lumelskii, Y., Pensky, M., (2003). The Stress-Strength Model and its Generalizations: Theory and Applications. Singapore: World scientific.

14. Lindley, D.V., (1980). Approximate Bayesian method. Trabajos de Estadistica, 31, 223237. 
15. Makhdoom, I., Nasiri, P., Pak, A., (2016). Bayesian approach for the reliability parameter of power Lindley distribution. International Journal of System Assurance Engineering and Management, 7(3), 341-355.

16. Nadar, M. and Kizilaslan, F., (2016). Estimation of reliability in a multicomponent stressstrength model based on a Marshall-Olkin bivariate Weibull distribution. IEEE Transactions on Reliability, 65(1), 370-380.

17. Pak, A. and Dey, S., (2019). Statistical Inference for the power Lindley model based on record values and inter-record times. Journal of Computational and Applied Mathematics, 347, 156-172.

18. Pak, A., Parham, G.H., Saraj, M., (2014a). Inferences on the Competing Risk Reliability Problem for Exponential Distribution Based on Fuzzy Data. IEEE Transactions on Reliability 63(1), 1-10.

19. Rao, G.S. (2012), Estimation of reliability in multicomponent stress-strength model based on generalized exponential distribution. Revista Colombiana de Estadistica, 35(1), 67-76.

20. Rao, G.S., Aslam, M. and Kundu, D., (2014). Burr Type XII distribution parametric estimation and estimation of reliability in multicomponent stress-strength model, Communication in Statistics-Theory and Methods, 44(23), 4953-4961.

21. Raqab, M.Z., Bdair, O.M. and Al-Aboud, F.M., (2018). Inference for the two-parameter bathtub-shaped distribution based on record data. Metrika, 81(3), 229-253.

22. Selim, M.A., (2018). Estimation and prediction for Nadarajah-Haghighi distribution based on record values. Pakistan Journal of Statistics 34(1), 77-90.

23. Tarvirdizade, B., and Ahmadpour, M., (2016). Estimation of the stress-strength reliability for the two-parameter bathtub-shaped lifetime distribution based on upper record values. Statistical Methodology, 31, 58-72.

24. Wu, J.W., Wu, C.C., and Tsai, M.H., (2005). Optimal parameter estimation of the twoparameter bathtub-shaped lifetime distribution based on a type II right censored sample. Applied Mathematics and Computation, 167, 807-819. 